\title{
In silico analysis of a novel causative mutation in Cadherin23 gene identified in an Omani family with hearing loss
}

\author{
Mohammed Nasser Al-Kindi ${ }^{1}$, Mazin Jawad Al-Khabouri ${ }^{2}$, Khalsa Ahmad Al-Lamki ${ }^{1}$, Flavia Palombo ${ }^{3}$, \\ Tommaso Pippucci ${ }^{3}$, Giovanni Romeo ${ }^{3}$ and Nadia Mohammed Al-Wardy ${ }^{1 *}$ (i)
}

\begin{abstract}
Background: Hereditary hearing loss is a heterogeneous group of complex disorders with an overall incidence of one in every 500 newborns presented as syndromic and non-syndromic forms. Cadherin-related 23 (CDH23) is one of the listed deafness causative genes. It is found to be expressed in the stereocilia of hair cells and in the retina photoreceptor cells. Defective CDH23 have been associated mostly with prelingual severe-to-profound sensorineural hearing loss (SNHL) in either syndromic (USH1D) or non-syndromic SNHL (DFNB12) deafness. The purpose of this study was to identify causative mutations in an Omani family diagnosed with severe-profound sensorineural hearing loss by whole exome sequencing technique and analyzing the detected variant in silico for pathogenicity using several in silico mutation prediction software.
\end{abstract}

Results: A novel homozygous missense variant, c.A7436C (p. D2479A), in exon 53 of CDH23 was detected in the family while the control samples were all negative for the detected variant. In silico mutation prediction analysis showed the novel substituted D2479A to be deleterious and protein destabilizing mutation at a conserved site on $\mathrm{CDH} 23$ protein.

Conclusion: In silico mutation prediction analysis might be used as a useful molecular diagnostic tool benefiting both genetic counseling and mutation verification. The aspartic acid 2479 alanine missense substitution might be the main disease-causing mutation that damages $\mathrm{CDH} 23$ function and could be used as a genetic hearing loss marker for this particular Omani family.

Keywords: $\mathrm{CDH} 23$, d2484a, In silico, Oman

\section{Background}

With the high rate of consanguineous marriages, several inherited diseases have been diagnosed among the Arab population including syndromic and non-syndromic deafness. A survey conducted in 2016 indicated that up to $49 \%$ of Omani marriages were consanguineous [1]. As a result, $70 \%$ of hearing loss cases in Oman were reported as possible inherited forms and until now, two genes have been reported to be involved in nonsyndromic autosomal recessive genetic deafness in Oman, MYO15A, and Otoferlin [2-4].

\footnotetext{
* Correspondence: naiwardi@squ.edu.om

'Department of Biochemistry, College of Medicine and Health Sciences,

Sultan Qaboos University, P.O. Box 35, Al-Khoud, 123 Muscat, Oman

Full list of author information is available at the end of the article
}

There are no exact statistical figures of syndromic or non-syndromic hearing loss in Oman. However, worldwide studies revealed that approximately 466 million people $(5.0 \%)$ of the world's population were suffering from hearing loss [5]. Earlier studies highlighted that about $30 \%$ of the total deafness cases are syndromic [6]. Usher syndrome (USH) is one of the syndromic deafness forms with an estimated prevalence of 1 in 6000 to 1 in 10,000 representing about $6 \%$ of the total congenital deafness and approximately $50 \%$ of hereditary deaf-blind individuals. USH is a genetic disorder accompanied by a dual sensory impairment, sensorineural hearing loss, retinitis pigmentosa, and variable vestibular dysfunction. Clinically, it is categorized into three subclasses: USH1, USH2, and USH3. USH1 is the most severe form, characterized by congenital severe to profound deafness, vestibular 
dysfunction, and prepubertal onset of visual loss. It accounts for 33 to $44 \%$ of USH cases. USH2 impact has been ranked from moderate to severe hearing loss with no vestibular dysfunction. It affects 56 to $67 \%$ of all USH patients. USH3 is less severe and is characterized by progressive hearing and vestibular function loss. It is found in 1 to $6 \%$ in the general population. However, in the Finnish and Ashkenazi Jews populations, it rises to about $40 \%$ [7-10]. So far, 13 genes have been identified to be involved in USH development (https://sph.uth.edu/ Retnet/sum-dis.htm). Among these genes is cadherinrelated 23 (CDH23), causing Usher syndrome type 1D (USH1D) [11, 12]. Studies revealed that a defective $\mathrm{CDH} 23$ gene plays an important role in developing Usher syndrome (OMIM \#601067) where it accounts for up to $32 \%$ of USH1 cases [13]. More than 350 associated mutations have been reported as either homozygous nonsense, frame-shift, splice-site, or missense mutations [14, 15]. Defective CDH23 was also detected in autosomal recessive non-syndromic hearing loss (OMIM \#601386) (DFNB12) where more than 24 associated mutations have been reported as missense mutations [16-19]. In the cell membrane, CDH23 interacts with procadherin 15 (PCDH15) to create stereocilia organization and hair bundle formation which reflects its importance in normal inner-ear mechanotransduction [20].

Next generation sequencing (NGS) made a big leap in genome DNA sequencing. A whole exome and a gene panel can be rapidly sequenced, and the abnormality and specificity of the genome can be detected in a short period. However, the Sanger principle remains a useful technique for sequencing a short DNA fragment and for the confirmation of the NGS findings.

In this study, we genetically analyzed an Omani family diagnosed clinically with severe to profound hearing loss. $\mathrm{Mu}$ tation detection was performed by Illumina HiSeq2000 platform (Illumina Inc., San Diego, CA, USA) NGS technique. DNA of an affected family member was sequenced to identify the family-specific mutated gene loci. The Sanger sequencing technique (ABI $3130 \mathrm{xl}$ ) was then applied for the whole family and control samples to confirm the NGS findings. A homozygous missense mutation in exon 53 of the cadherin-related 23 gene $(\mathrm{CDH} 23)$ was detected in all affected members but was absent in the normal family members and controls. Subsequently, in silico genetic testing was used to verify the pathogenicity of the identified mutation.

\section{Methods}

This study was conducted by the Department of Biochemistry, College of Medicine and Health Sciences, Sultan Qaboos University, and the Department of ENT, Al Nahdha Hospital, Ministry of Health, Oman, with collaboration from the Medical Genetics Unit, Polyclinic Sant'Orsola-Malpighi, Bologna, Italy.

Clinical examination: Four affected members, two males and two females, from one Omani family of consanguineous marriage (degree of parental relatedness, first cousins) were enrolled in this study.

Clinical history and audiological evaluation were done at the ENT department, Al Nahdha Hospital. Clinical examination was conducted using standard pure tone audiometry (PTA), optoacoustic and acoustic emittance

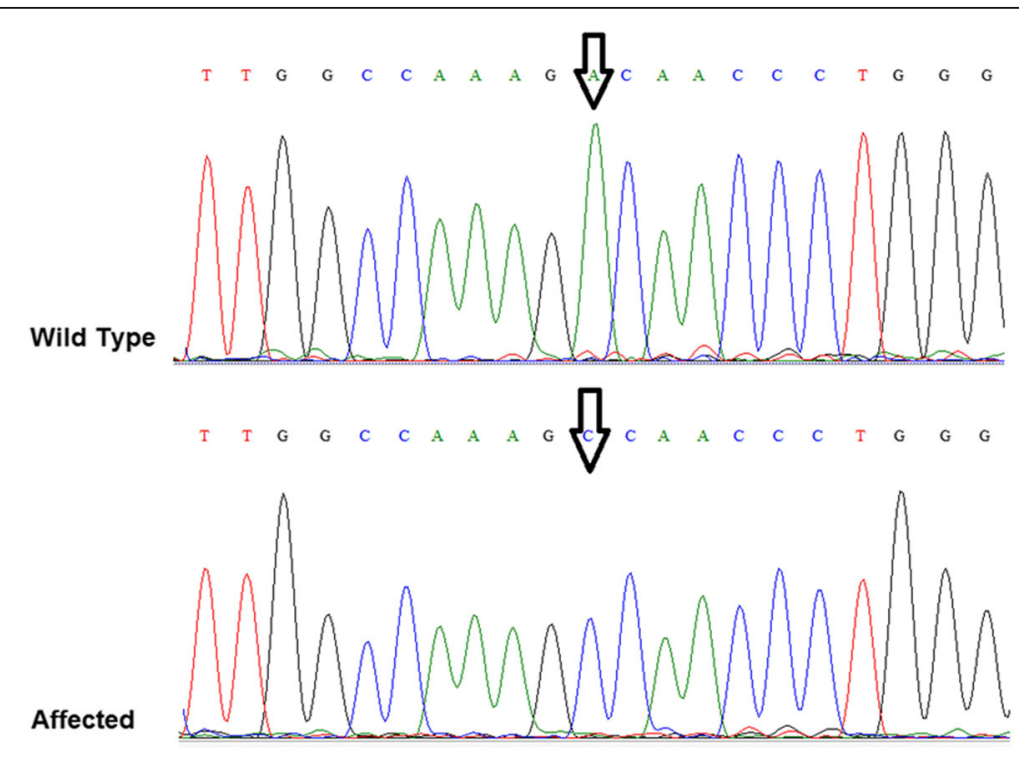

Fig. 1 Electropherogram of $\mathrm{CDH} 23$ gene mutation position. Wild-type sequence represents normal control sample with AA genotype. Affected represents a deaf family member with CC genotype 


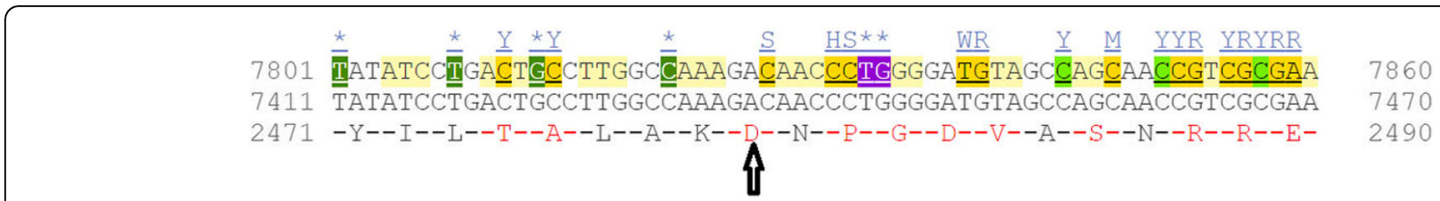

Fig. 2 Normal cDNA of CDH23 transcript containing GAC (aspartic acid)

tests. Blood samples from patients and their close relatives were collected in EDTA tubes. Samples from 130 male and female individuals without any hearing or visual disorders were used as normal controls.

DNA extraction and sequencing: Qiagen kit (Qiagen, Hilden, Germany) was used to extract the genomic DNA from peripheral blood of all collected samples. DNA from an affected member was analyzed using the Illumina HiSeq2000 platform (Illumina Inc., San Diego, CA, USA) NGS technique. The Sanger sequencing method (ABI PRISM Big-Dye terminator cycle sequencing premix kit (PE Applied Biosystems, Austin, TX, USA) was used to sequence 442 base pairs including the variant site to confirm the NGS finding. The rest of the family members and 130 controls were also tested for the detected variant. Polymerase chain reaction (PCR) forward (5'TCAGTGTCAAATCTCCAGAG3') and reverse (5'TTGGCAAAGATTTCTCCCAG3') primers were designed to amplify and confirm the NGS detected variant.

In silico analysis: In order to evaluate the putative pathological nature of the detected missense variant, physical properties of the amino acids such as change in hydrophobicity, and the impact of the substitution on protein structure and function, were analyzed using the online software SIFT (http://sift.jcvi.org/www/SIFT_enst_submit.html) [21], Mutation Taster (http://www.mutationtaster.org) [22], Mutation Assessor (http://mutationassessor.org/r3/) [23], PolyPhen-2 (http://genetics.bwh.harvard.edu/pph2/) [24], $\quad$ Mutpred2 (http://mutpred2.mutdb.org/index.html) [25], and SNAP2 (https://rostlab.org/services/snap2web/) [26]. Additional

Table 1 Online in silico analysis tools used for $\mathrm{CDH} 23$ mutation pathogenicity prediction

\begin{tabular}{ll}
\hline Tool & Prediction value \\
\hline SIFT & 0.00 \\
Polyphen & 1.000 \\
Provean & -5.48 \\
MutPred2 & 0.854 \\
SNAP2 & 19 \\
Mutation Assessor & Fl score 4.485 \\
Mutation Taster & Causing disease (0.99)
\end{tabular}

A variant is considered to be deleterious and damaging if the predicted values are as follows: SIFT $>0.05$ (damaging), Polyphen $<0.8$ (probably damaging), Provean $>-2.5$ (deleterious), MutPred $2<0.5$ (deleterious), SNAPs $<0.00$ nonneutral (deleterious), Mutation Assessor $<3.5$ (high-functional, Fl score 3.505.50), Mutation Taster $<0.5$ (disease-causing) software such as MUpro (http://mupro.proteomics.ics.uci. edu/) [27], I-Mutant 2.0 (http://folding.biofold.org/i-mutant/imutant2.0.html) [28], and DUET (http://biosig.unimelb.edu. $\mathrm{au} /$ duet/) [29] were used to predict the impact of the mutation on protein structure stability based on the change in Gibbs free energy $(\Delta \Delta G)$.

The protein secondary structure was visualized and annotated using the online POLYVIEW (http://polyview. cchmc.org/) [30]. RMSD (root-mean-square deviation) and

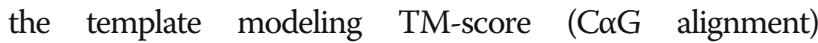
(https://zhanglab.ccmb.med.umich.edu/TM-score/tmp/33 9254.html) were used to calculate the atomic deviation and the degree of similarity by superimposing models of native and mutant proteins [31, 32]. The variant genetics database including its location verification on the whole genome, cDNA, and protein sequences were retrieved by using the NCBI (National Center for Biological Information) (http://www.ncbi.nlm.nih.gov) and Ensemble genome browser (https://asia.ensembl.org/) online programs [33]. PrimerZ (http://genepipe.ncgm.sinica.edu.tw/ primerz/ beginDesign.do) and BioEdit (http://www.mbio. ncsu.edu/ BioEdit/bioedit.html) were used for DNA primer design and sequence alignment [34,35]. Validation of the protein degree of activity and function, the residues percentage of distribution of three regions, favored, allowed and residue in outlier region were analyzed and calculated using Ramachandran plot and the online software RAMPAGE (http://mordred.bioc.cam.ac.uk/ rapper/rampage.php) [36]. Attribution of the residue position to the protein function was analyzed by SWISS-MODEL template library (http:// swissmodel.expasy. org) and visualized by SwissPDBViewer [37], Chimera program (https://www.cgl.ucsf. edu/chimera/download.html) [38], and Jmol (http://jmol. sourceforge.net) [39]. The retrieved variant was subjected

Table 2 Online in silico analysis tools used for $\mathrm{CDH} 23$ protein stability changes and protein folding similarities

\begin{tabular}{ll}
\hline Tool & Prediction value \\
\hline MUpro & DDG $=-0.58$ \\
I-Mutant 2.0 (kcal/mol) & DDG $=-1.22$ \\
DUET $(\mathrm{kcal} / \mathrm{mol})$ & $\mathrm{DDG}=-2.88$ \\
TM-score & 0.99786 \\
RMSD $(\AA)$ & 0.44 \\
\hline
\end{tabular}

MUpro, I-Mutant 2.0, and DUET: DDG increases in protein stability $>0>$ decrease in stability. $0.5<\mathrm{TM}$-score $<1.00$ reflects that the status of protein folding is identical. RMSD $0 \AA-2 \AA$ reflects perfect match alignment 


Mutated
VTATDAD SGNFALIEY SLGDGESKFAINPTTGDIY VL SSLDREKKDHYILTAL AKANPGDV ASNRRENSVQVVIQVLDVN
DCRP.
Normal
VTATDAD SGNFALIEY SLG DGESKFAINPTTGDIY VLSSLDREKKDHYILTAL AKDNPGDV ASNRRENSVQVVIQVLDVN
DCRP

Fig. 3 Mutated and normal CA-domain 23 amino acid sequence of CDH23. D2484A substitution is shown in the mutated (A) and normal (D) domain

to further analysis to predict the rate of conservation by alignment with close living species by using the ConSurf Server analysis package (http://consurf.tau.ac.il/2016/) [40], NCBI multiple sequence alignment (MSA) (https://www. ebi.ac.uk/Tools/msa/muscle/) [41], and Crustal Omega (http://www.ebi.ac.uk/Tools/msa/clustalo/) [42].

\section{Results}

\section{DNA sequencing}

The genetic abnormality of the affected family members diagnosed with hearing loss was detected by next generation sequencing whole exome technology. A novel homozygous missense variant, g.A71800709C, c.A7436

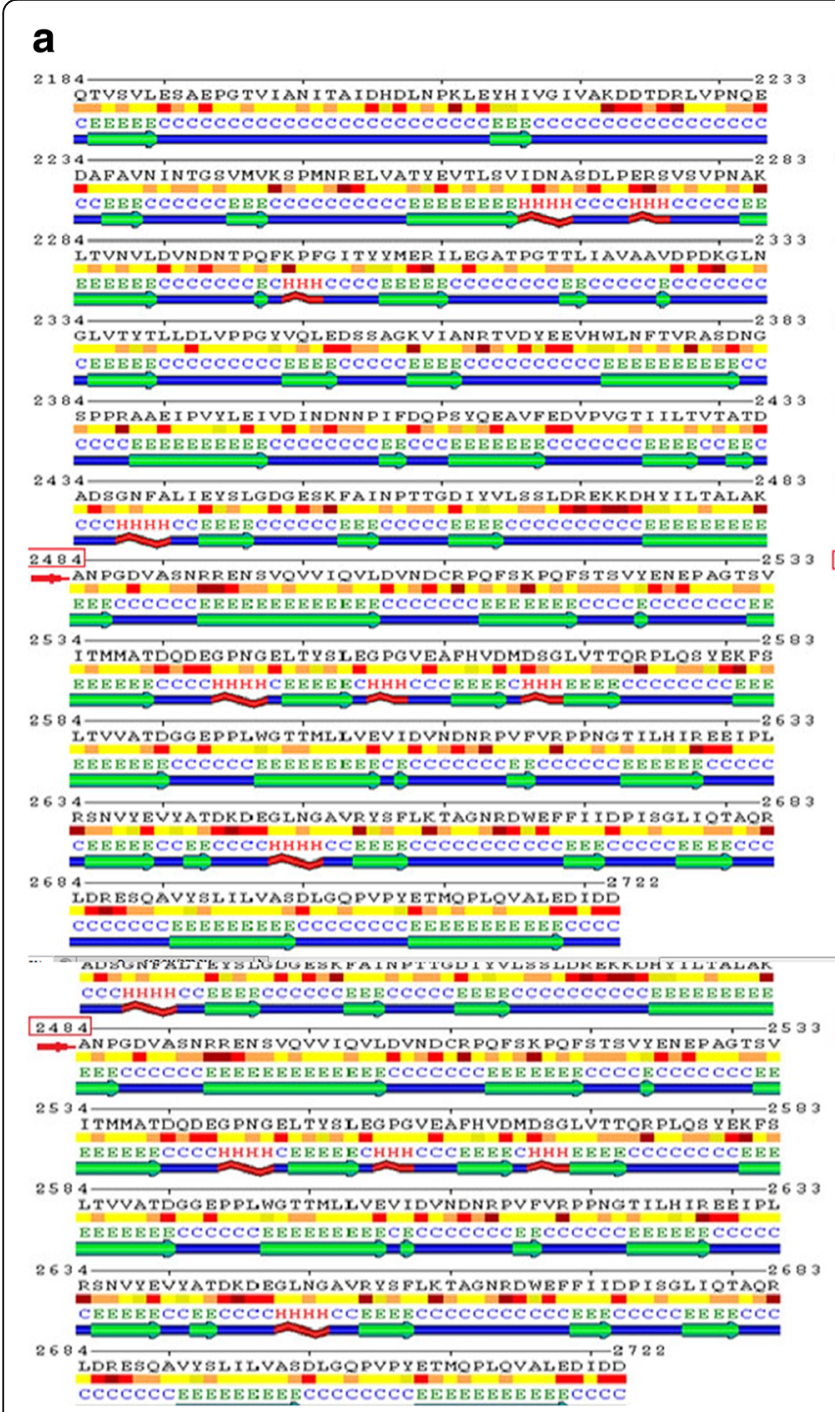

b

2184 QTVSVLESAEPGTVIANI IA IDHDINPKLEYH IVG IVAKDDTDRLVPNOE CEEEEECCCCCCCCCCCCCCCCCCCCCCCEERCCCCCCCCCCCCCCC $2 2 3 4 \longdiv { \text { DAFAVN INTGSVMVKSPMNRELVATYEVTLSVIDNASDLPERSVSVPNAK } } 2 8 3$ CCEEECCCCCCEEECCCCCCCCCCEEEEEEEEHHHCCCCHHHCCCCCE CCEEECCCCCCEECCCCCCCCCCEEEEEEEHHHHCCCCHHHCCCCCEE 284 TVNVLDVNDNTPQFKPFG ITYYMER ILEGATPGTTLIAVAAVDPDKGLN 233 EEEEEECCCCCCCECHHHCCCCEEEEEECCCCCCEEECCCCECCCCCCC $2 3 3 4 \longdiv { \text { GLYTYTLLDLVPPG YVQ́LEDSSAGKVI ANRTVDYEEVHWLNFTVRASDNG } } 2 3 8$ CEEEEECCCCCCCCCEEEECCCCCEEEECCCCCCCCCCEEEEEEEEECC 2384 PPRAAEI PVYLEIVDINDNN I FDQPS YQEAVFEDVPVGTIILTVTATD 243 CCCCEEEEEEEEEECCCCCCCEECCCEEEEEEECCCCCCCEEEECCEEC 434 DSGNFAL IEYSLGDGESKFAINPTTGDIXVLSSLDREKKDHXILTALAK 243 ADSGNFALTEYSLGDGESKFATNPTTGDTXVLSSLDREKKDHXILTALA 2484 - DNPGDVASNRRENSVQVVIQVLDVNDCRPQFSK PQFSTSVYENEPAGTS CCCCCCCCCCCEEEEEEEEEECCCCCCCEEEEEEECCCCECCCCCCCEE $2 5 3 4 \longdiv { \text { TMMATDODEG PNGELTXSLEG PGVEÁRHVDMDSGLVTTQRPLQS XEKFS } } 2 5 3$ EEEEEECCCCCCCCCEEEEECHHHCCCEEECCCCCEREECCCCCCCCEE

$2584 \overbrace{\text { TVVATDGGEPPLWGTTMLLVEVIDVNDNRPVFVRPPNGTILHIREEIPL }}^{2} 633$ EEEEEEECCCCCCEEEEEEEEECECCCCCCCEECCCCCCEEEEEECCCCC

$2 6 3 4 \longdiv { \text { RSNVYEVYATDKDEGLNG AVR YSFLKTAGNRDWEFFIIIDPISGL IOTAOR } } 2 6$ CEN CEEEEECCEECCCCCCCCCEEEECCCCCCCHHHHEEECCCCCEEEECCC

$2 6 8 4 \longdiv { \text { DRESQÁVYSLILVASDLGQPVPXETMOPLQVALEDIDD } } 2 2 2$ CCCCCCCEEEEEEEEECCCCCCCCEEEEEEEEEEECCCC ADSGNFAL IEXSLGDGESKFAINPTTGDIXVLSSLDREKKDHXILTALAK CCCHHHHCCEEEECCCCCCEEECCCCCEEEECCCCCCCCCCEEEEEEEEE

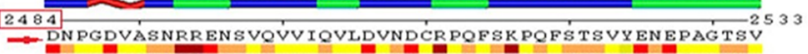
CCCCCCCCCCCEEEEEEEEEECCCCCCCEEEEEEECCCECCCCCCCE

$5 3 4 \longdiv { \text { TMMATDODEG PNGELTYSLEG PGVEÁFHVDMDSGLVTTORPLQS XEKFS } } 2 5 8$ EEEEEECCCCCCCCCEEEECHHHCCCEEECCCCCEEEECCCCCCCCEEE

$2584{ }_{\text {TVVATDGGEPPLWGTTMLLVEVIDVNDNRPVFVRPPNGTILHIREEIPL }}^{2} 633$ EEEEEEECCCCCCEEEEEEEEECECCCCCCCEECCCCCCEEEEEECCCCC

$2 6 3 4 \longdiv { \text { RSNVYEVYATDKDEGLNGAVRYSFLKTAGNRDWEFFIIDPISGLIQTAQR } } 2 6 3$ CEEEEECCEECCCCCCCCCCEEEECCCCCCCHHHHEEECCCCERECC $=0$

$2 6 8 4 \longdiv { \text { DRESQÁVYSLILVASDLGQPVPYETMQPLQVALEDIDD } } 2 7 2$ CCCCCCCEEEEEEEECCCCCCCCEEEEEEEEECCCC

Fig. 4 Secondary structure of $\mathrm{CDH} 23$ model-template alignment (5szn.1.A). a 2484 ALA predicted within beta strand. b 2484 ASP predicted within coiled coil. The red color indicates the highest level of amino acid conservation, while yellow indicates the lowest 
$\mathrm{C}$, replacing the negatively charged aspartic acid residue with a nonpolar aliphatic amino acid alanine at position D 2479A in exon 53 of CDH23 gene was confirmed and verified by Sanger sequencing (Fig. 1).

The CDH23 gene located on chromosome 10 contains 70 exons as illustrated on ensemble protein transcript CDH23 ENST00000224721.12, ENSG00000107736, Pfam: PF00028, and UniProtKBA0A0A0MQS6. Figure 2 illustrates the normal cDNA of $\mathrm{CDH} 23$ transcript as obtained from Ensemble genomic browser.

The CDH23 transcript (A0A0A0MQS6) was selected for further analysis to identify functional protein domains using the online SMART program. The program detected one signal peptide, 26 cadherin repeats (CA), also known as extracellular cadherin (EC) domains, one transmembrane region, and one low complexity region. The variant was found on domain 23 of the 26 CA (Fig. 2).

\section{In silico mutation analysis}

The detected missense point variant was evaluated for its pathogenicity using different mutation prediction programs (Table 1) and was considered to be deleterious and damaging.

The impact of the variant on protein stability changes was studied to evaluate its leverage on protein folding. The unfolding Gibbs free energy change (DDG or $\Delta \Delta G$ ) was calculated using MUpro, I-Mutant 2.0, and DUET online tools. Models of native and mutant proteins were superimposed to predict the level of similarity between the two protein structures using the template modeling score (TM-score) and the root-mean-square deviation (RMSD) online software (Table 2).

The domain of interest was further analyzed for secondary structure prediction. Polyview-2D was used to predict the possible effects of the detected variant on the confirmation of domain 23. The impact of amino acid exchange on domain structure was evaluated by comparing wild-type predicted secondary structures and mutant sequences. The mutated domain structure was predicted to consist of 292 coils, 218 strands, and 28 helices compared to 302 coils, 215 strands, and 21 helices in the wild type (Fig. 3).

CDH23 transcript (A0A0A0MQS6) for the wild and mutated types was analyzed by the Swiss model program to build up possible protein templates and models. 5szn.1.A was selected to be the template and building model for $\mathrm{CDH} 23$ because of its similar identity with the wild and mutated types (33.96 and 34.52\%, respectively). The D2479A ensemble position moved to D2484A on the 5szn model. Jmol package and Ramachandran plots were used to align and validate the two 3D structures in order to predict the possible impact of the mutated

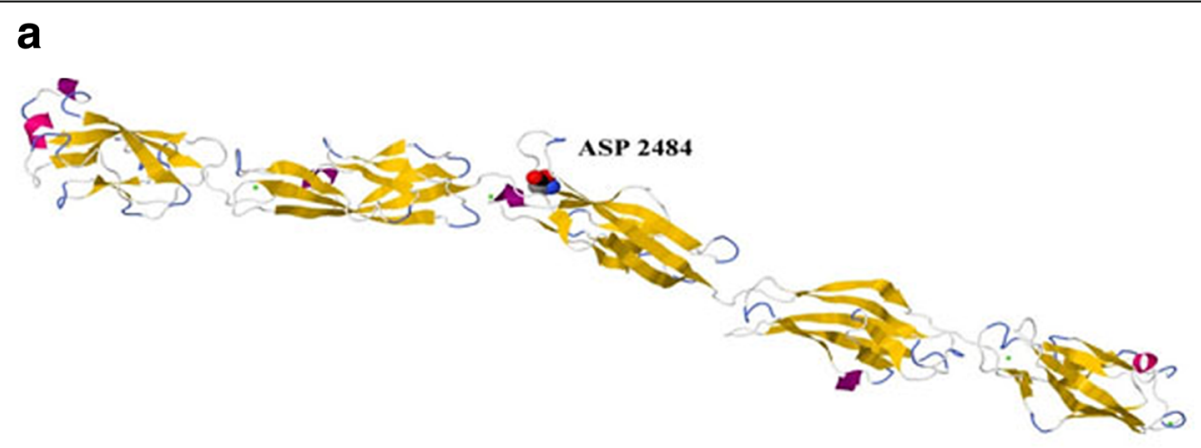

b

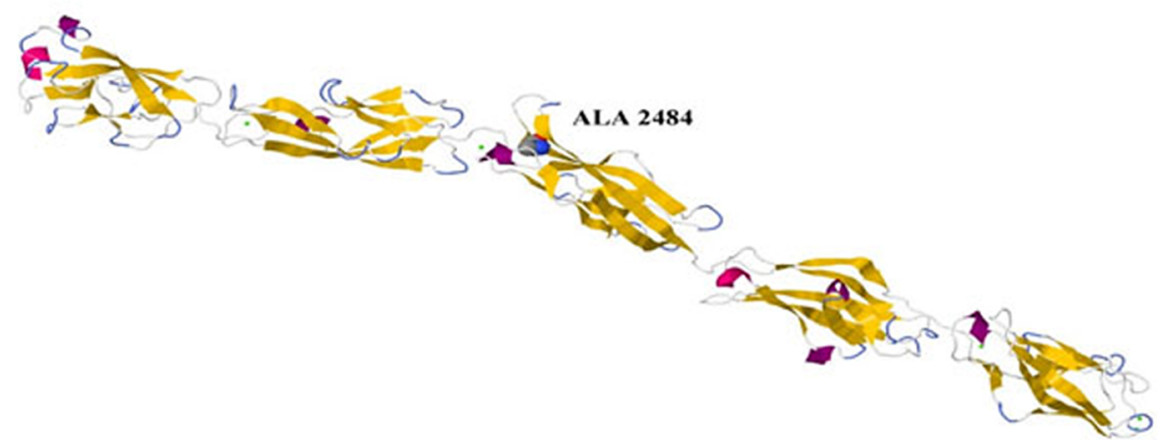

Fig. 5 Molecular modeling of wild-type and mutant $\mathrm{CDH} 23$. Location of $\mathbf{a}$ the wild-type ASP (aspartic acid) as a part of the B strand and $\mathbf{b}$ the mutated ALA (Alanine) as a part of the turning loop of the extracellular cadherin (EC) domain 23 of CDH23 model 
amino acid on $\mathrm{CDH} 23$ protein structure (Figs. 4, 5, 6, and 7).

The Ramachandran plot was used to calculate and visualize the dihedral angles predicting the energetically allowed residues based upon their phi and psi dihedral angles. A score of $\geq 90 \%$ in the allowed regions shows that the built model has high quality (Fig. 8 and Table 3).

The evolutionary conservation rate of the substitution was analyzed using the online NCBI protein cluster (Fig. 9) and ConSurf programs (Fig. 10).

\section{Discussion}

$\mathrm{CDH} 23$ is an adhesive protein expressed in the neurosensory epithelium of the inner ear hair cells and encodes the transmembrane $\mathrm{Ca}^{2+}$-dependent adhesion protein, cadherin 23 ( $\mathrm{CDH} 23)$ [43]. It is thought to be involved in stereocilia organization and hair bundle formation [44]. Using its adhesion property, it interacts with protocadherin 15 protein to form a tip-link filamentous complex, which is the main component that drives the normal mechano-transduction process in auditory and vestibular hair cells. Hence, a change in the protein structure might lead to a significant defect in its comprehended performance, which, in turn, could terminate the entire inner ear mechano-transduction process by turning off the sound perception and acceleration stages. The impact of the defective $\mathrm{CDH} 23$ protein was observed in both syndromic and non-syndromic hearing loss forms $[11,43]$. It accounted for up to $32 \%$ of Usher syndrome type 1 cases [13]. More than 24 associated mutations have been reported as missense mutations that clearly appeared as an important cause of hearing loss in Asian populations [16-19]. Recent research studies suggest that in silico mutation prediction might be used as a first-line molecular diagnosis tool serving both genetic counseling and mutation verification and variant classification $[45,46]$. Prediction of variant pathogenicity using bioinformatics tools was conducted by several studies. A homozygous c.5985C > A (p.Y1995X) variant, a heterozygous p.E1006K, and p.D1663V were detected in the Chinese population [47, 48]. The mutation frequency spectrum of $\mathrm{CDH} 23$ among the recessive inherited cases is $5.7 \%$ in the Japanese population and $15 \%$ in the Korean population [16, 19, 49]. Other gene variants were also analyzed using such programs such as V66 M variant of human BDNF in psychiatric disorders and computational modeling of complete HOXB13 protein for predicting the functional effect of SNPs and the associated role in hereditary prostate cancer $[50,51]$. The American College of Medical Genetics and Genomics (ACMG) guide for the interpretation of sequence variants
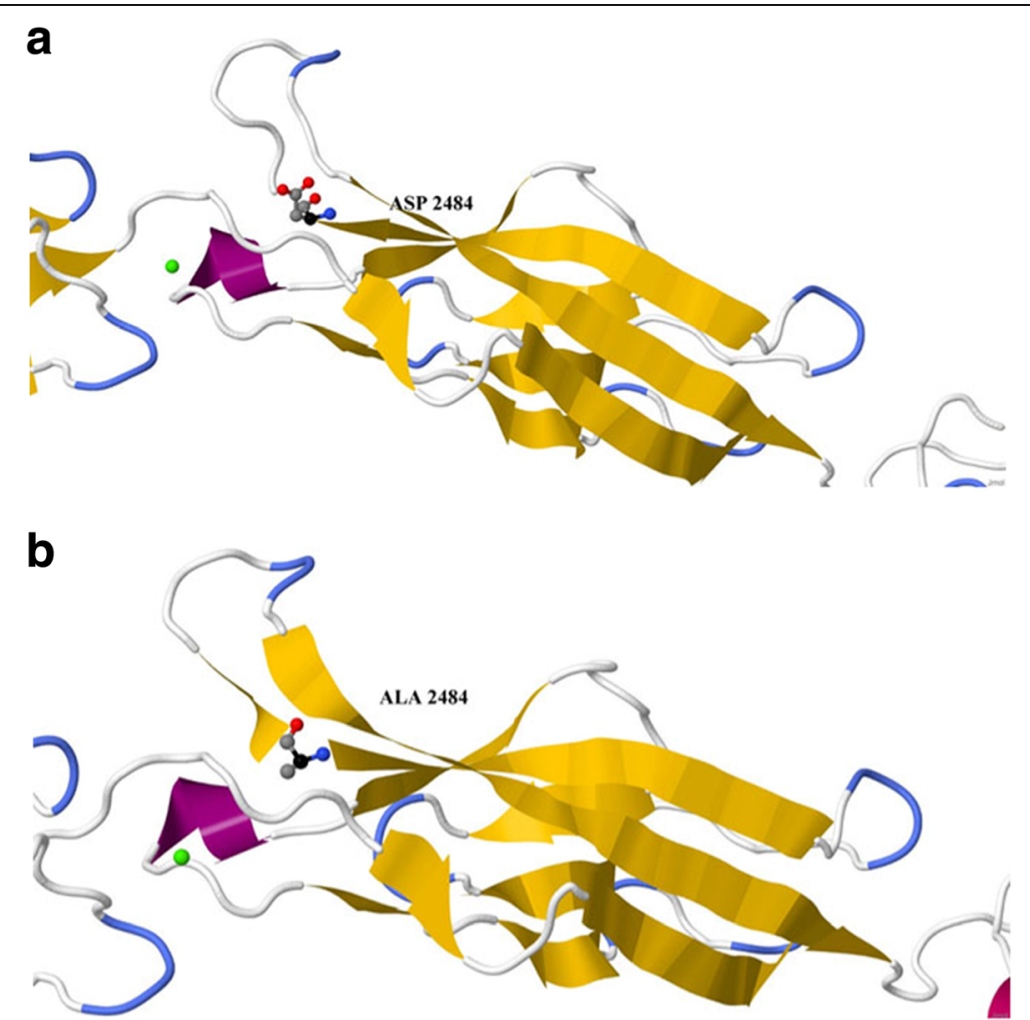

Fig. 6 Magnified EC23 and the location of a ASP and $\mathbf{b}$ ALA of CDH23 model. Calcium molecules (red balls) are clearly seen as part of the structure 

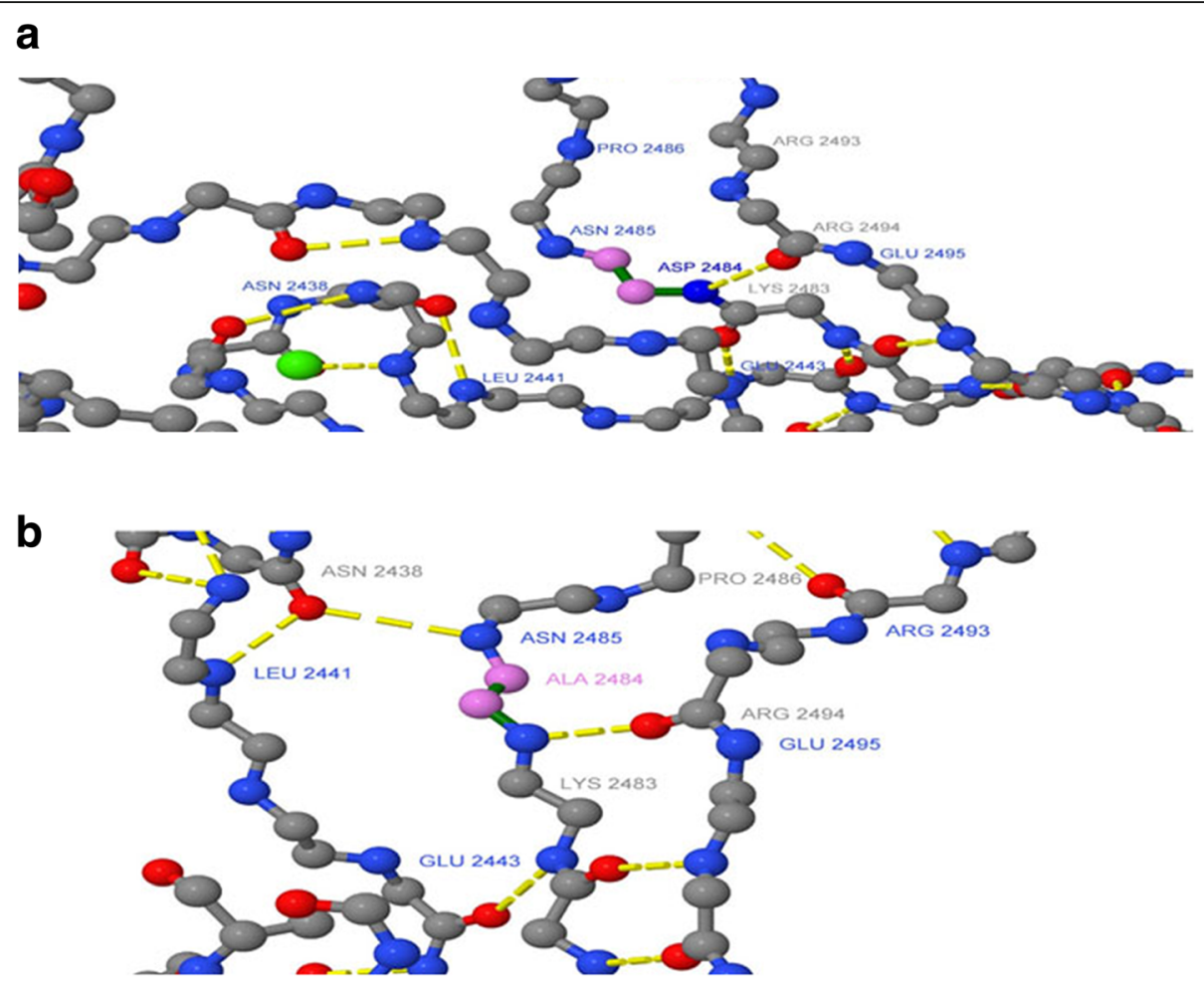

Fig. 7 Predicted crystal native and mutant protein secondary structures. Interaction of CA 23 amino acids to form the CDH23 secondary structure. a The position of the native ASP 2484 and $\mathbf{b}$ the mutated ALA 2484. Hydrogen bonds are indicated with yellow lines

elaborated the usefulness of the predictive software programs for risk estimation and accurate interpretation of the potential causality of sequence variation [52]. The variant specifications (location), classification (mutation type), and pathogenicity degree interpretation (pathogenic, likely pathogenic, uncertain significance, likely benign, and benign) were thoroughly revised by the ACMG, and the use of specific standard terminology in describing the variant identity was recommended $[45,46]$.

In this study, we genetically analyzed an Omani family who was diagnosed clinically with severe to profound hearing loss. The analysis revealed a missense variant on

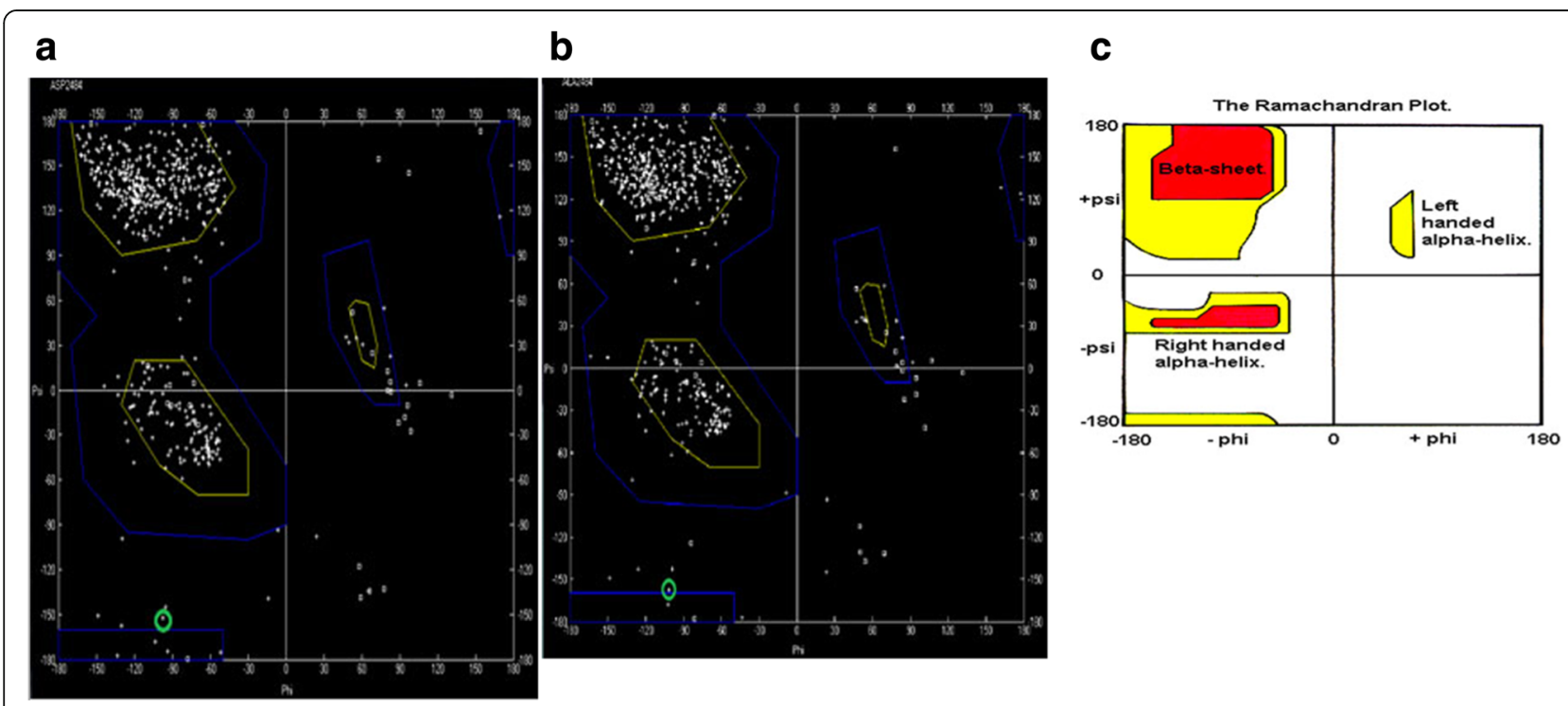

Fig. 8 The Ramachandran plot for the $\mathrm{CDH} 232484$ residue. a Native CDH23 with ASP at position 2848. b Mutant CDH23 with ALA at position 2484. c Normal distribution of Ramachandran plot 
Table 3 RAMPAGE: assessment of the Ramachandran plot

\begin{tabular}{|c|c|c|c|c|c|c|}
\hline \multirow[t]{2}{*}{ Model } & \multicolumn{2}{|c|}{$\begin{array}{l}\text { Residues in favored } \\
\text { regions }\end{array}$} & \multicolumn{2}{|c|}{$\begin{array}{l}\text { Residues in allowed } \\
\text { regions }\end{array}$} & \multicolumn{2}{|c|}{$\begin{array}{l}\text { Residue in outlier } \\
\text { region }\end{array}$} \\
\hline & $\begin{array}{l}\text { No. of } \\
\text { residues }\end{array}$ & $\begin{array}{l}\% \text { of } \\
\text { residues }\end{array}$ & $\begin{array}{l}\text { No. of } \\
\text { residues }\end{array}$ & $\begin{array}{l}\% \text { of } \\
\text { residues }\end{array}$ & $\begin{array}{l}\text { No. of } \\
\text { residues }\end{array}$ & $\begin{array}{l}\% \text { of } r \\
\text { esidues }\end{array}$ \\
\hline Wild-type residue [A2484:ASP] $(-97.36,-152.49)$ in allowed region & 505 & 94.0 & 25 & 4.7 & 7 & 1.3 \\
\hline Mutant-type residue $[$ A2484:ALA] $(-101.70,-157.59)$ in allowed region & 506 & 94.2 & 24 & 4.5 & 7 & 1.3 \\
\hline
\end{tabular}

CDH23 (c.A7436C) which was detected by NGS technology and confirmed by Sanger DNA sequencing methodology. Affected family members had the CC genotype, while all 120 normal control samples had the wild-type genotype of AA. The identified variant was subjected to various in silico functional prediction algorithm software to evaluate the pathogenicity level, functionality, and protein stability. The evaluation was based on criteria such as variant location on the genome, sequence homology, conservation level, and physicochemical and structural properties. Sorting Intolerant from Tolerant (SIFT), Polymorphism Phenotyping 2 (PolyPhen 2), PROVEAN (Protein Variation Effect Analyzer), MutPred2 (Mutation Prediction), screening for non-acceptable polymorphisms (SNAPs), and Mutation Assessor protein function prediction software were used, and all agreed on the pathogenicity of the variant. The results from these predictors classified the variant as damaging, deleterious, and disease-causing, and boosted the variant damaging level on the mutant protein structure.

Prediction of the variant's influence on the stability of protein structure is a crucial aspect for studying the function of the protein. The unfolding Gibbs free energy change $(\Delta G)$ of the native and mutant structures was calculated by subtracting the free energy change of the mutant protein from the free energy change of the native protein $(\mathrm{Kcal} / \mathrm{mol}$ ) (DDG or $\Delta \Delta G)=\Delta G$ mutant $-\Delta G$ wild type. Above zero value of DDG predicts high stability of the mutant protein and a score below zero predicts low stability [53, 54]. Structure stability was predicted by using I-Mutant 2.0, MUpro, and DUET programs. All analyses agreed that variant p.D2479A might destabilize the protein structure by indicating a negative score.

Alignment and proteins similarity are important factors assessing generated protein models of related identity. The template modeling score (Tm-score) was used to determine the topological deviation of native from mutant model structures, whereas RMSD was used to calculate the average distance of the alpha carbon backbones between the two models [55]. Both programs predicted a perfect match between the two model structures-wild type and mutant. The sequence of amino acids determines the protein conformation, and the physical and chemical properties of the amino acids greatly affects protein function. Alanine, known as a strong helix-favoring residue, engages in van der Waals interactions, nonpolar and uncharged (hydrophobic) status. Aspartic acid, on the other hand, is negative in charge, polar, and able to make hydrogen bonds with other amino acids and water (hydrophilic status). The substitution in this case might change the protein self-interaction. Therefore, secondary and tertiary protein structures were further analyzed to investigate the impact of the mutant variant on the protein function. According to the Polyview-2D results, the mutated domain structure was predicted to consist of 292 coils, 218 strands, and 28 helices compared to 302 coils, 215 strands, and 21 helixes in the wild type. It is clearly seen that alanine is located within the $\beta$-strand segment of the mutated protein whereas aspartic acid is located within the coiled loop of the wild-type protein [56, 57]. Templates and models for both proteins were created by

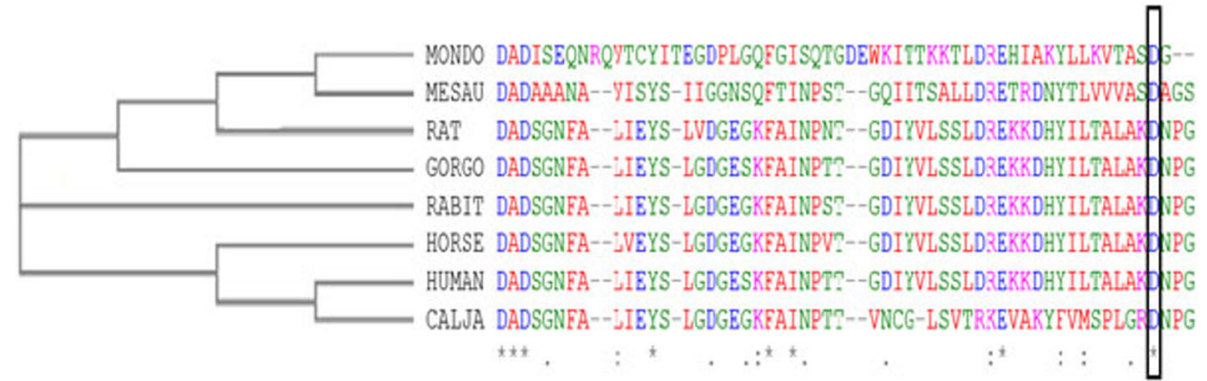

Fig. 9 Conservation analysis of aspartic acid 2479 residue. CA 23 of Human CDH23 alignment with other mammalian protein sequences. Phylogenetic tree and MSA sequence alignment of ASP (the black block) degree of conservation of Human and other mammalian species 


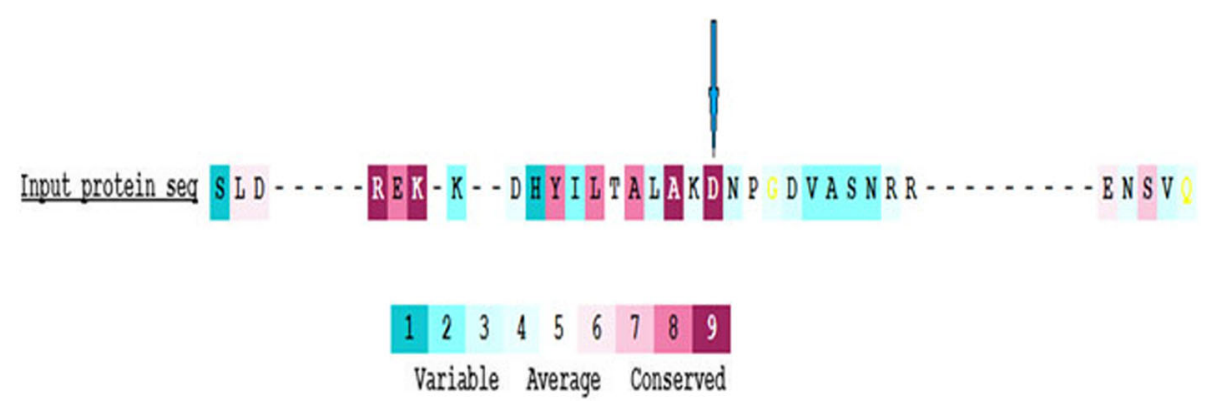

Fig. 10 The position of ASP (D) predicted to be within the highest conserved region with a value of 9

using the Swiss model online program. The complete protein structure of the $\mathrm{CDH} 23$ protein was not available in the Protein Data Bank (PDB). Hence, PDB files for both mutant and native proteins were modeled by Swiss model online program. The two models supported the Polyview2D findings. PDB files were analyzed and visualized by SWISS PDB Viewer and Jmol. The difference in hydrogen bonding on the native and mutant protein domain was calculated. Both native and mutant variants were hydrogen-bonded with ARG 2494. However, asparagine at position 2485 bonded with asparagine at position 2438 in the mutated but not in the wild-type form. This could indicate that changing the amino acid aspartate to alanine could affect protein structure and, therefore, its function. CDH23 gene information was retrieved from NCBI, Ensemble genome browser, and UniProt database. Analysis showed that the detected variant is a novel homozygous missense variant located at g. 71800709, c.A7436C, p.D2479A, chr10:71800709, q22.1 in exon 53, of ENST00000224721 of GRCh38.p7. The exact location of variant domain on the protein was predicted using Smart genetics program and was found to be within the CAdomain 23. The domain consists of 84 amino acids ordered from 2424 to 2507 and is reported as cadherin repeat in the extracellular domain of a transmembrane $\mathrm{CDH} 23$ [58]. The total number of repeats is 27 presented within the adherent junctions region as a glycoprotein. The EC domains are involved in cell-to-cell adhesion via hemophilic calcium-dependent interactions [59]. Binding of calcium to the EC domains at the linker region between consecutive EC repeats promotes the linearization, rigidity, and dimerization of $\mathrm{CDH} 23$ [60]. The aspartic acid residues have a high $\mathrm{Ca}^{+}$affinity, and that may play an important role in the interactions of $\mathrm{CDH} 23$ molecules either with $\mathrm{CDH} 23$ or with other proteins. Since calcium provides rigidity to the elongated structure of cadherin molecules and enables hemophilic lateral interaction, the mutation is likely to result in a decreased affinity for calcium and, in turn, impairs the whole process of protein interaction [61]. A Phi/Psi two torsion angles $\mathrm{N}-\mathrm{C} \alpha$ (called Phi, $\phi)$ and $C \alpha-C$ (called Psi, $\psi$ ) in a polypeptide chain play a role in the control of local structure folding. Therefore, applying Ramachandran plot would predict the protein folding capability and, in turn, predict the quality of the three-dimensional structures. A Ramachandran plot was obtained to validate the protein structures that were created by the Swiss model for both mutant and native template models. Swiss PDB viewer was used to create Ramachandran plot, and Rampage program was used to calculate the amino acid assembly point percentage. According to the program, a good protein structure model is expected to have more than $90 \%$ of the residues within the core or favored region of the protein. RAMPAGE predicted greater than $94 \%$ of the 537 residues assembled within the favored region of both native and mutant proteins [62]. Conserved amino acids in proteins are found to be involved in various cellular processes in a biological system including genome stability [63]. Due to this, phylogeny and multi-sequencing alignment (MSA) were conducted to evaluate the aspartic acid 2484 stability and conservation status. As was predicted by ConSurf Server package and Polyview-2D, aspartic acid is highly conserved with a score of 9 among species reflecting the importance of this amino acid position that may play a crucial role in the integrity of protein structure and conformation.

One limitation of this study is that the detected mutation was identified by next generation sequencing technology, which requires sequencing of the whole human exon. The technique is outside the routine daily assays, and the running cost is high. However, the mutation confirmation assay by Sanger DNA sequencing technology is more economical.

\section{Conclusion}

In this study, we used various in silico mutation prediction programs to analyze a substituted variant on $\mathrm{CDH} 23$ protein. The variant was typed on an Omani family diagnosed clinically with hearing loss. The analysis predicted the novel substituted D2479A to be deleterious and protein destabilizing mutation at a conserved site on $\mathrm{CDH} 23$ protein. This mutation might lead to a major disruption in 
CDH23 protein structure that may cause disturbance of stereocilia organization and hair bundle formation affecting the mechano-transduction process and, in turn, hearing loss. In silico mutation prediction analysis might be used as a useful molecular diagnostics tool benefiting both genetic counseling and mutation verification in the governmental and private sectors. The affected family might benefit from the outcome of this research by considering the potential risk of consanguineous marriage.

\section{Abbreviations}

ALA: Alanine; ASP: Aspartic acid; CDH23: Cadherin-related 23; DDG or $\Delta \Delta \mathrm{G}$ : Gibbs free energy change; $\mathrm{EC}$ : Extracellular; MSA: Multiple sequence alignment; MutPred2: Mutation prediction; NCBl: National Center for Biological Information; NGS: Next generation sequencing;

PCDH15: Procadherin 15; PolyPhen 2: Polymorphism phenotyping 2; PROVEAN: Protein Variation Effect Analyzer; PTA: Pure tone audiometry; RMSD: Root-mean-square deviation; SIFT: Sorting Intolerant from Tolerant: SNAPS: Screening for non-acceptable polymorphisms; SNHL: Sensorineural hearing loss; TM-score: Template modeling score; USH: Usher syndrome

\section{Acknowledgements}

The authors wish to thank the Omani family members who participated in this research.

The abstract has been presented at ICMBH 2018: International Conference on Molecular Biology of Hearing held from January 25-26, 2018, in Paris, France.

\section{Authors' contributions}

MNK and NMW designed the study and analyzed and validated the data. MNK performed the experiments and wrote the manuscript. KAL, FP, and TP performed the experiments and analyzed the data. MJK provided the blood samples and clinical data. GR revised the manuscript, and NMW edited and revised the manuscript and approved the final version. All the authors read and approved the manuscript.

\section{Funding}

This study was financially supported by Sultan Qaboos University, 2017, grant number IG/MED/BIOC/17/01. The funding body did not participate in the design of the study, nor in the collection, analysis, and interpretation of data or in writing the manuscript.

\section{Availability of data and materials}

All data generated or analyzed during this study are included in this published article.

\section{Ethics approval and consent to participate}

This work was approved by the College of Medicine and Health Sciences Ethics Committee, Ref. No. SQU-EC/121/16, MREC \#1311. Written consent was obtained from all study participants.

\section{Consent for publication}

Not applicable.

\section{Competing interests}

The authors declare that they have no competing interests.

\section{Author details}

'Department of Biochemistry, College of Medicine and Health Sciences, Sultan Qaboos University, P.O. Box 35, Al-Khoud, 123 Muscat, Oman. ${ }^{2}$ Department of Otolaryngology and Head and Neck Surgery, Al Nahda Hospital, Ministry of Health, Muscat, Oman. ${ }^{3}$ Medical Genetics Unit, Polyclinic Sant'Orsola-Malpighi, University of Bologna, Bologna, Italy.
Received: 3 October 2019 Accepted: 19 January 2020

Published online: 02 March 2020

\section{References}

1. Mazharul IM (2017) Consanguineous marriage in Oman: understanding the community awareness about congenital effects of and attitude towards consanguineous marriage. Hum Biol 44:273-286. https://doi.org/10.1080/ 03014460.2016.1224385

2. Al-Wardy NM, Al-Kindi MN, Al-Khabouri MJ, Tamimi Y, Van Camp G (2016) A novel missense mutation in the C2C domain of otoferlin causes profound hearing impairment in an Omani family with auditory neuropathy. Saudi Med J 37:1068-1075. https://doi.org/10.15537/smj.2016.10.14967

3. Khabori MA, Patton MA (2008) Consanguinity and deafness in Omani children. Int J Audiol 47:30-33. https://doi.org/10.1080/14992020701703539

4. Palombo F, Al-Wardy N, Ruscone GAG, Oppo M, Kindi MNA, Angius A et al (2017) A novel founder MYO15A frameshift duplication is the major cause of genetic hearing loss in Oman. J Hum Genet 62:259-264. https://doi.org/ 10.1038/jhg.2016.120

5. Schmucker C, Kapp P, Motschall E, Loehler J, Meerpohl JJ (2019) Prevalence of hearing loss and use of hearing aids among children and adolescents in Germany: a systematic review. BMC Public Health 18(19):1277. https://doi. org/10.1186/s12889-019-7602-7

6. Van Camp G, Willems PJ, Smith RJ (1997) Nonsyndromic hearing impairment: unparalleled heterogeneity. Am J Hum Genet 60:758-764

7. Hope Cl, Bundey S, Proops D, Fielder AR (1997) Usher syndrome in the city of Birmingham_-prevalence and clinical classification. Br J Ophthalmol 81:46-53

8. Kimberling WJ, Hildebrand MS, Shearer AE, Jensen ML, Halder JA, Trzupek K et al (2010) Frequency of Usher syndrome in two pediatric populations: implications for genetic screening of deaf and hard of hearing children. Genet Med 12:512-516. https://doi.org/10.1097/GIM.0b013e3181e5afb8

9. Koffler T, Ushakov K, Avraham KB (2015) Genetics of hearing loss. Otolaryngol Clin N Am 48:1041-1061. https://doi.org/10.1016/j.otc.2015.07.007

10. Sun T, Xu K, Ren Y, Xie Y, Zhang X, Tian L et al (2018) Comprehensive molecular screening in Chinese Usher syndrome patients. Invest Opthalmol Vis Sci 59:1229. https://doi.org/10.1167/iovs.17-23312

11. Bork JM, Peters LM, Riazuddin S, Bernstein SL, Ahmed ZM, Ness SL et al (2001) Usher syndrome 1D and nonsyndromic autosomal recessive deafness DFNB12 are caused by allelic mutations of the novel cadherin-like gene CDH23. Am J Hum Genet 68:26-37. https://doi.org/10.1086/316954

12. Bolz H, von Brederlow B, Ramírez A, Bryda EC, Kutsche K, Nothwang HG et al (2001) Mutation of $\mathrm{CDH} 23$, encoding a new member of the cadherin gene family, causes Usher syndrome type 1D. Nat Genet 27:108-112. https://doi.org/10.1038/83667

13. Oshima A, Jaijo T, Aller E, Millan JM, Carney C, Usami S et al (2008) Mutation profile of the $\mathrm{CDH} 23$ gene in 56 probands with Usher syndrome type I. Hum Mutat 29:E37-E46. https://doi.org/10.1002/humu.20761

14. Bolz HJ, Roux A-F (2011) Clinical utility gene card for: Usher syndrome. Eur J Human Genet 19. https://doi.org/10.1038/ejhg.2011.15

15. Valero R, de Castro-Miró M, Jiménez-Ochoa S, Rodríguez-Ezcurra JJ, Marfany G, Gonzàlez-Duarte R (2019) Aberrant splicing events associated to CDH23 noncanonical splice site mutations in a proband with atypical Usher syndrome 1. Genes 10. https://doi.org/10.3390/genes10100732

16. Miyagawa M, Nishio S, Usami S (2012) Prevalence and clinical features of hearing loss patients with $\mathrm{CDH} 23$ mutations: a large cohort study. PLoS One 7:e40366. https://doi.org/10.1371/journal.pone.0040366

17. Schultz JM, Bhatti R, Madeo AC, Turriff A, Muskett JA, Zalewski CK et al (2011) Allelic hierarchy of $\mathrm{CDH} 23$ mutations causing non-syndromic deafness DFNB12 or Usher syndrome USH1D in compound heterozygotes. J Med Genet 48:767-775. https://doi.org/10.1136/jmedgenet-2011-100262

18. Vanniya SP, Chandru J, Pavithra A, Jeffrey JM, Kalaimathi M, Ramakrishnan $R$ et al (2018) Recurrence of reported CDH23 mutations causing DFNB12 in a special cohort of south Indian hearing impaired assortative mating families_an evaluation. Ann Hum Genet 82:119-126. https://doi.org/10. 1111/ahg.12228

19. Woo H-M, Park H-J, Park M-H, Kim B-Y, Shin J-W, Yoo WG et al (2014) Identification of $\mathrm{CDH} 23$ mutations in Korean families with hearing loss by whole-exome sequencing. BMC Med Genet 15:46. https://doi.org/10.1186/ 1471-2350-15-46

20. Alagramam KN, Goodyear RJ, Geng R, Furness DN, van Aken AFJ, Marcotti W et al (2011) Mutations in protocadherin 15 and cadherin 23 affect tip 
links and mechanotransduction in mammalian sensory hair cells. PLoS One 6:e19183. https://doi.org/10.1371/journal.pone.0019183

21. Ng PC, Henikoff S (2003) SIFT: predicting amino acid changes that affect protein function. Nucleic Acids Res 31:3812-3814

22. Schwarz JM, Rödelsperger C, Schuelke M, Seelow D (2010) MutationTaster evaluates disease-causing potential of sequence alterations. Nat Methods 7: 575-576. https://doi.org/10.1038/nmeth0810-575

23. Adzhubei IA, Schmidt S, Peshkin L, Ramensky VE, Gerasimova A, Bork P et al (2010) A method and server for predicting damaging missense mutations. Nat Methods 7:248-249. https://doi.org/10.1038/nmeth0410-248

24. Ramensky V, Bork P, Sunyaev S (2002) Human non-synonymous SNPs: server and survey. Nucleic Acids Res 30:3894-3900. https://doi.org/10.1093/nar/gkf493

25. Li B, Krishnan VG, Mort ME, Xin F, Kamati KK, Cooper DN et al (2009) Automated inference of molecular mechanisms of disease from amino acid substitutions. Bioinformatics (Oxford, England) 25:2744-2750. https://doi.org/ 10.1093/bioinformatics/btp528

26. Hecht $M$, Bromberg $Y$, Rost B (2015) Better prediction of functional effects for sequence variants. BMC Genomics 16(Suppl 8):S1. https://doi.org/10. 1186/1471-2164-16-S8-S1

27. Cheng J, Randall A, Baldi P (2006) Prediction of protein stability changes for single-site mutations using support vector machines. Proteins 62:1125-1132. https://doi.org/10.1002/prot.20810

28. Capriotti E, Calabrese R, Casadio R (2006) Predicting the insurgence of human genetic diseases associated to single point protein mutations with support vector machines and evolutionary information. Bioinformatics (Oxford, England) 22:2729-2734. https://doi.org/10.1093/ bioinformatics/bt/423

29. Pires DEV, Ascher DB, Blundell TL (2014) DUET: a server for predicting effects of mutations on protein stability using an integrated computational approach. Nucleic Acids Res 42:W314-W319. https://doi. org/10.1093/nar/gku411

30. Porollo AA, Adamczak R, Meller J (2004) POLYIEW: a flexible visualization tool for structural and functional annotations of proteins. Bioinformatics (Oxford, England) 20:2460-2462. https://doi.org/10.1093/bioinformatics/bth248

31. Martí-Renom MA, Stuart AC, Fiser A, Sánchez R, Melo F, Sali A (2000) Comparative protein structure modeling of genes and genomes. Annu Rev Biophys Biomol Struct 29:291-325. https//doi.org/10.1146/annurev.biophys.29.1.291

32. Zhang Y, Skolnick J (2005) TM-align: a protein structure alignment algorithm based on the TM-score. Nucleic Acids Res 33:2302-2309. https://doi.org/10. 1093/nar/gki524

33. Wheeler DL, Church DM, Edgar R, Federhen S, Helmberg W, Madden TL et al (2004) Database resources of the National Center for biotechnology information: update. Nucleic Acids Res 32:D35-D40. https://doi.org/10.1093/ nar/gkh073

34. Tsai M-F, Lin Y-J, Cheng Y-C, Lee K-H, Huang C-C, Chen Y-T et al (2007) PrimerZ: streamlined primer design for promoters, exons and human SNPs. Nucleic Acids Res 35:W63-W65. https://doi.org/10.1093/nar/gkm383

35. Thomas PD, Campbell MJ, Kejariwal A, Mi H, Karlak B, Daverman R et al (2003) PANTHER: a library of protein families and subfamilies indexed by function. Genome Res 13:2129-2141. https://doi.org/10.1101/gr.772403

36. Lovell SC, Davis IW, Arendall WB, de Bakker PIW, Word JM, Prisant MG et al (2003) Structure validation by Calpha geometry: phi,psi and Cbeta deviation. Proteins 50:437-450. https://doi.org/10.1002/prot.10286

37. Guex N, Peitsch MC (1997) SWISS-MODEL and the Swiss-Pdb Viewer: an environment for comparative protein modeling. Electrophoresis 18:27142723. https://doi.org/10.1002/elps.1150181505

38. Schwede T, Kopp J, Guex N, Peitsch MC (2003) UCSF chimera-a visualization system for exploratory research and analysis. Nucleic Acids Res 31:3381-3385

39. Herráez A (2006) Biomolecules in the computer: Jmol to the rescue. Biochem Mol Biol Educ 34:255-261. https://doi.org/10.1002/bmb.2006. 494034042644

40. Ashkenazy H, Erez E, Martz E, Pupko T, Ben-Tal N (2010) ConSurf 2010: calculating evolutionary conservation in sequence and structure of proteins and nucleic acids. Nucleic Acids Res 38:W529-W533. https://doi.org/10. 1093/nar/gkq399

41. Edgar RC (2004) MUSCLE: multiple sequence alignment with high accuracy and high throughput. Nucleic Acids Res 32:1792-1797. https://doi.org/10. 1093/nar/gkh340

42. Sievers F, Wilm A, Dineen D, Gibson TJ, Karplus K, Li W et al (2011) Fast, scalable generation of high-quality protein multiple sequence alignments using Clustal Omega. Mol Syst Biol 7:539. https://doi.org/10. 1038/msb.2011.75

43. Astuto LM, Bork JM, Weston MD, Askew JW, Fields RR, Orten DJ et al (2002) $\mathrm{CDH} 23$ mutation and phenotype heterogeneity: a profile of 107 diverse families with Usher syndrome and nonsyndromic deafness. Am J Hum Genet 71:262-275. https://doi.org/10.1086/341558

44. Siemens J, Lillo C, Dumont RA, Reynolds A, Williams DS, Gillespie PG et al (2004) Cadherin 23 is a component of the tip link in hair-cell stereocilia. Nature 428:950-955. https://doi.org/10.1038/nature02483

45. Richards S, Aziz N, Bale S, Bick D, Das S, Gastier-Foster J et al (2015) Standards and guidelines for the interpretation of sequence variants: a joint consensus recommendation of the American College of Medical Genetics and Genomics and the Association for Molecular Pathology. Genet Med 17: 405-424. https://doi.org/10.1038/gim.2015.30

46. Parsamanesh N, Safarpour H, Etesam S, Shadmehri AA, Miri-Moghaddam E (2019) Identification and in silico characterization of a novel point mutation within the phosphatidylinositol glycan anchor biosynthesis class $\mathrm{G}$ gene in an Iranian family with intellectual disability. J Mol Neurosci 69:538-545. https://doi.org/10.1007/s12031-019-01376-y

47. Chai Y, Chen D, Sun L, Li L, Chen Y, Pang X et al (2015) The homozygous $p$. V37I variant of GJB2 is associated with diverse hearing phenotypes. Clin Genet 87:350-355. https://doi.org/10.1111/cge.12387

48. Lu Y, Zhou X, Jin Z, Cheng J, Shen W, Ji F et al (2014) Resolving the genetic heterogeneity of prelingual hearing loss within one family: performance comparison and application of two targeted next generation sequencing approaches. J Hum Genet 59:599-607. https://doi.org/10.1038/jhg.2014.78

49. Xu T, Zhu W, Wang P, Li H, Yu S (2019) Identification of novel cadherin 23 variants in a Chinese family with hearing loss. Mol Med Rep 20:2609-2616. https://doi.org/10.3892/mmr.2019.10503

50. Chandrasekaran G, Hwang EC, Kang TW, Kwon DD, Park K, Lee J-J et al (2017) Computational modeling of complete HOXB13 protein for predicting the functional effect of SNPs and the associated role in hereditary prostate cancer. Sci Rep 7:43830. https://doi.org/10.1038/srep43830

51. De Oliveira CCS, Pereira GRC, De Alcantara JYS, Antunes D, Caffarena ER, De Mesquita JF (2019) In silico analysis of the V66M variant of human BDNF in psychiatric disorders: an approach to precision medicine. PLoS One 14: e0215508. https://doi.org/10.1371/journal.pone.0215508

52. Richards CS, Bale S, Bellissimo DB, Das S, Grody WW, Hegde MR et al (2008) ACMG recommendations for standards for interpretation and reporting of sequence variations: revisions 2007. Genet Med 10:294-300. https://doi.org/ 10.1097/GIM.0b013e31816b5cae

53. Mavroconstanti T, Johansson S, Winge I, Knappskog PM, Haavik J (2013) Functional properties of rare missense variants of human $\mathrm{CDH} 13$ found in adult attention deficit/hyperactivity disorder (ADHD) patients. PLoS One 8 : e71445. https://doi.org/10.1371/journal.pone.0071445

54. Nailwal M, Chauhan JB (2017) In silico analysis of non-synonymous single nucleotide polymorphisms in human DAZL gene associated with male infertility. Syst Biol Reprod Med 63:248-258. https://doi.org/10.1080/ 19396368.2017 .1305466

55. Arshad M, Bhatti A, John P (2018) Identification and in silico analysis of functional SNPs of human TAGAP protein: A comprehensive study. PLoS One 13:e0188143. https://doi.org/10.1371/journal.pone.0188143

56. Uslupehlivan $M$, Sener E, Deveci R (2018) In silico analysis of Pax6 protein glycosylation in vertebrates. Comput Biol Chem 77:116-122. https://doi.org/ 10.1016/j.compbiolchem.2018.09.016

57. Fazel-Najafabadi E, Vahdat Ahar E, Fattahpour S, Sedghi M (2015) Structural and functional impact of missense mutations in TPMT: An integrated computational approach. Comput Biol Chem 59 Pt A:48-55. https://doi.org/ 10.1016/..compbiolchem.2015.09.004

58. Ortiz FW, Sergeev $\mathrm{YV}$ (2019) Global computational mutagenesis of domain structures associated with inherited eye disease. Sci Rep 9:3676. https://doi. org/10.1038/s41598-019-39905-9

59. Angst BD, Marcozzi C, Magee Al (2001) The cadherin superfamily: diversity in form and function. J Cell Sci 114:629-641

60. Nagar B, Overduin M, Ikura M, Rini JM (1996) Structural basis of calciuminduced E-cadherin rigidification and dimerization. Nature 380:360-364. https://doi.org/10.1038/380360a0

61. de Brouwer APM, Pennings RJE, Roeters M, Van Hauwe P, Astuto LM, Hoefsloot LH et al (2003) Mutations in the calcium-binding motifs of $\mathrm{CDH}_{2} 3$ and the 35delG mutation in GJB2 cause hearing loss in one family. Hum Genet 112:156-163. https://doi.org/10.1007/s00439-002-0833-0 
62. Khan SH, Javed MR, Qasim M, Shahzadi S, Jalil A, Rehman SU (2014) Domain analyses of Usher syndrome causing Clarin-1 and GPR98 protein models. Bioinformation 10:491-495. https://doi.org/10.6026/97320630010491

63. Greene LH, Chrysina ED, Irons LI, Papageorgiou AC, Acharya KR, Brew K (2001) Role of conserved residues in structure and stability: tryptophans of human serum retinol-binding protein, a model for the lipocalin superfamily. Protein Sci 10:2301-2316. https://doi.org/10.1110/ps.22901

\section{Publisher's Note}

Springer Nature remains neutral with regard to jurisdictional claims in published maps and institutional affiliations.

Submit your manuscript to a SpringerOpen ${ }^{\circ}$ journal and benefit from:

- Convenient online submission

Rigorous peer review

- Open access: articles freely available online

High visibility within the field

- Retaining the copyright to your article

Submit your next manuscript at $\boldsymbol{\nabla}$ springeropen.com 